\title{
System Dynamics Control through the Fractal Potential
}

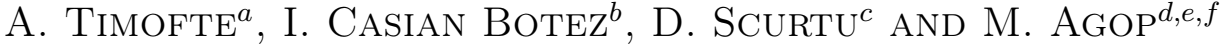 \\ ${ }^{a}$ Faculty of Physics, "Al. I. Cuza" University, Iasi 700050, Romania \\ ${ }^{b}$ Faculty of Electronics and Telecomunications, "Gh. Asachi" Technical University, Iasi 700514, Romania \\ ${ }^{c}$ Department of Fluid Mechanics, "Gh. Asachi" Technical University, Iasi 700514, Romania \\ ${ }^{d}$ Department of Physics, University of Athens, Athens 15771, Greece \\ ${ }^{e}$ Laboratoire de Physique des Lasers, Atomes et Molécules, Centre d'Etudes et de Recherches Lasers \\ et Applications, Université Lille 1 Sciences et Technologies, 59655 Villeneuve d'Ascq Cedex, France \\ ${ }^{f}$ Department of Physics, "Gh. Asachi" Technical University, Iasi 700514, Romania \\ (Received June 12, 2010; revised version November 2, 2010; in final form November 15, 2010)
}

\begin{abstract}
Implications of the fractal potential in the system dynamics using an extended scale relativity model assuming the fractal character of the particle movements, are established. So, in the dissipative approximation of the model it is shown that the fractal potential comes from the non-differentiability of the space-time, i.e. by means of imaginary part of a complex speed field. In the dispersive approximation of the same model, the fractalization of the differential part of the complex speed field induces a normalized fractal potential which controls through coherence the system dynamics. In such context the type I superconductivity results: the temperature dependences of the superconducting parameter, the accumulator effect etc.
\end{abstract}

PACS: 05.45.Df, 47.53. $+\mathrm{n}, 03.65 .-\mathrm{w}$

\section{Introduction}

A way to analyze the dynamics of the physical systems is to consider that the particle movements take place on fractals (the complexity of these dynamics is substituted by fractality) [1-6]. There are some arguments which can justify such hypothesis: (i) by interaction, the trajectory is no longer everywhere differentiable. The "uncertainty" in tracking the particle is eliminated by means of the fractal approximation of motion; (ii) the complex dynamical systems, which display chaotic behaviour, are recognized to acquire self-similarity and manifest strong fluctuations at all possible scales [7-12]. Every type of "elementary" process of motion induces both spatio-temporal scales and the associated fractals. Moreover, the movement complexity is directly related to the fractal dimension: the fractal dimension increases as the movement becomes more complex [1]. Different definitions were given for the fractal dimension (the Kolmogorov dimension, the Hausdorff dimension etc. [1-5]), but once we choose the fractal-type dimension in the study of motion we must work with it until the end.

Therefore, considering that the complexity of the physical processes (from the system's interactions) is replaced by fractality (situation in which the particle movements take place on fractal curves), it is no longer necessary to use notions as collision time, mean free path, etc., i.e., the whole classical "arsenal" of quantities from the dynamics of physical systems. Then, the physical systems will behave as a special interaction-less "fluid" by means of geodesics in a fractal space-time. The theory which treats the interactions in the previously mentioned man- ner is the scale relativity (SR) $[5,9-11,13-22]$. It is based on a generalization of Einstein's principle of relativity to scale transformations. Namely, "one redefines space-time resolutions as characterizing the state of reference systems scale, in the same way as speed characterizes their state of motion. Then one requires that the laws of physics apply whatever the state of the reference system, of motion (principle of motion-relativity) and of scale (principle of SR). The principle of SR is mathematically achieved by the principle of scale-covariance, requiring that the equations of physics keep their simplest form under transformations of resolution" [5, 13].

The standard SR theory can be formulated both in the Schrödinger version and also in the hydrodynamic one considering that only the spatial coordinates are fractals, the time being an affine parameter. However, the hydrodynamic version implies the existence, at all resolution scale $[5,13]$, of a potential analog to the Bohm potential from the quantum mechanics [23-28]. We remind that the Bohm formulation of quantum mechanism in terms of quantum potential [23-28] has been and still is, a continuous matter of dispute, often in rather philosophical terms above all concerning the fundamental meaning of quantum mechanics. Regarding concrete applications, such a theory has been used as a theoretical tool for understanding and interpreting several processes in different fields [29-43], from molecular physics to plasma physics, from scattering theory to simulation of quantum wires $[44,45]$, to name a few.

Since the role of such potential was not sufficiently considered in the frame of an extended scale relativity 
(ESR) theory [46], the purpose of the present paper is to investigate its role in the system dynamics.

The plan of the paper is as follows: in Sect. 2 a short reminder on ESR is presented; in Sect. 3, through the dissipative approximation of the ESR, the expression of the fractal potential is established; in the dispersive approximation of the ESR, at non-differentiable scale, the fractal potential controls through coherence (accumulator effect) the system dynamics. Such situation is explicitly treated for type I superconductivity in Sect. 4.

\section{A short reminder on the extended scale relativity model}

Nottale suggests in his works the possibility to formulate the physical principles at different scales considering the geometry of space is continuous but non-differentiable, therefore it is fractalic (i.e., explicitly scale-dependent) $[5,9-11,13-22]$. His theory was mathematically substantiated by Ben Adda and Cresson's works [47-54]. Several generalizations of SR theory are given in $[29,34,46,55]$. The non-differentiability implies the followings:

(i) a continuous and a non-differentiable curve (or almost nowhere differentiable) is explicitly scale dependent, and its length tends to infinity, when the scale interval tends to zero. In other words, a continuous and non-differentiable space is fractal, in the general meaning given by Mandelbrot to this concept [1];

(ii) there is an infinity of fractals curves (geodesics) relating any couple of its points (or starting from any point), and this is valid for all scales;

(iii) the breaking of local differential time reflection invariance. The time-derivative of a function $F$ can be written twofold

$$
\begin{aligned}
\frac{\mathrm{d} F}{\mathrm{~d} t} & =\lim _{\mathrm{d} t \rightarrow 0} \frac{F(t+\mathrm{d} t)-F(t)}{\mathrm{d} t}, \\
\frac{\mathrm{d} F}{\mathrm{~d} t} & =\lim _{\mathrm{d} t \rightarrow 0} \frac{F(t)-F(t-\mathrm{d} t)}{\mathrm{d} t} .
\end{aligned}
$$

Both definitions are equivalent in the differentiable case. In the non-differentiable situation these definitions fail, since the limits are no longer defined. "In the framework of scale relativity, the physics is related to the behavior of the function during the "zoom" operation on the time resolution $\delta t$, here identified with the differential element $\mathrm{d} t$ ("substitution principle"), which is considered as an independent variable. The standard function $F(t)$ is therefore replaced by a fractal function $F(t, \mathrm{~d} t$ ) (for details see [47-54]) explicitly dependent on the time resolution interval, whose derivative is undefined only at the unobservable limit $\mathrm{d} t \rightarrow 0$ " $[5,9-11,13-22]$. As a consequence, this leads us to define the two derivatives of the fractal function as explicit functions of the two variables $t$ and $\mathrm{d} t$,

$$
\frac{\mathrm{d}_{+} F}{\mathrm{~d} t}=\lim _{\mathrm{d} t \rightarrow 0_{+}} \frac{F(t+\mathrm{d} t, \mathrm{~d} t)-F(t, \mathrm{~d} t)}{\mathrm{d} t},
$$

$$
\frac{\mathrm{d}_{-} F}{\mathrm{~d} t}=\lim _{\mathrm{d} t \rightarrow 0_{-}} \frac{F(t, \mathrm{~d} t)-F(t-\mathrm{d} t, \mathrm{~d} t)}{\mathrm{d} t} .
$$

The sign "+" corresponds to the forward process and "-" to the backward process;

(iv) the differential of a fractal function $F(t, \mathrm{~d} t)$ can be expressed as the sum of two differentials, one which is not scale-dependent, $\mathrm{d} F^{\prime}(t)$, and the other dependent on it, $\mathrm{d} F^{\prime \prime}(t, \mathrm{~d} t)$, therefore [47-54]:

$$
\mathrm{d} F(t, \mathrm{~d} t)=\mathrm{d} F^{\prime}(t)+\mathrm{d} F^{\prime \prime}(t, \mathrm{~d} t) .
$$

Particularly, the differential of the generalized coordinates, $\mathrm{d}_{ \pm} \boldsymbol{X}(t, \mathrm{~d} t)$, can be decomposed as follows:

$$
\mathrm{d}_{ \pm} \boldsymbol{X}(t, \mathrm{~d} t)=\mathrm{d}_{ \pm} \boldsymbol{x}(t)+\mathrm{d}_{ \pm} \boldsymbol{\xi}(t, \mathrm{~d} t)
$$

where $\mathrm{d}_{ \pm} \boldsymbol{x}(t)$ is the "classical part" and $\mathrm{d}_{ \pm} \boldsymbol{\xi}(t, \mathrm{~d} t)$ is the "fractal part". Starting from here, multiplying by $\mathrm{d} t^{-1}$ and using the substitutions

$$
\boldsymbol{V}_{ \pm}=\frac{\mathrm{d}_{ \pm} \boldsymbol{X}}{\mathrm{d} t}, \quad \boldsymbol{v}_{ \pm}=\frac{\mathrm{d}_{ \pm} \boldsymbol{x}}{\mathrm{d} t}, \quad \boldsymbol{u}_{ \pm}=\frac{\mathrm{d}_{ \pm} \boldsymbol{\xi}}{\mathrm{d} t},
$$

we obtain the velocity field

$$
\boldsymbol{V}_{ \pm}=\boldsymbol{v}_{ \pm}+\boldsymbol{u}_{ \pm}
$$

(v) the fractal part of $F$, i.e. $F^{\prime \prime}$, satisfies the relation

$$
\left|F^{\prime \prime}(t)-F^{\prime \prime}\left(t^{\prime}\right)\right| \approx\left|t-t^{\prime}\right|^{\delta},
$$

where $\delta$ depends on the fractal dimension $D_{\mathrm{F}}$ (for details see $[47-54])$.

Particularly, the differential of the "fractal part" of $\mathrm{d}_{ \pm} \boldsymbol{X}$, becomes

$$
\mathrm{d}_{ \pm} \xi_{i}=\sqrt{2 D}(\mathrm{~d} t)^{1 / D_{\mathrm{F}}}
$$

The significances of the Nottale coefficient $D$ [5] result from the random walk (Brownian motion) or its generalization, the Levy motion [5, 9-11, 13-22];

(vi) the local differential time reflection invariance is recovered by combining the two derivatives, $\mathrm{d}_{+} / \mathrm{d} t$ and $\mathrm{d}_{-} / \mathrm{d} t$, in the complex operator

$$
\frac{\hat{\mathrm{d}}}{\mathrm{d} t}=\frac{1}{2}\left(\frac{\mathrm{d}_{+}+\mathrm{d}_{-}}{\mathrm{d} t}\right)-\frac{\mathrm{i}}{2}\left(\frac{\mathrm{d}_{+}-\mathrm{d}_{-}}{\mathrm{d} t}\right) .
$$

We call this procedure "an extension by differentiability" (Cresson's extension — for details see [47-54]).

Applying this operator to the "position vector" yields a complex speed

$$
\begin{aligned}
\hat{\boldsymbol{V}} & =\frac{\hat{\mathrm{d}} \boldsymbol{X}}{\mathrm{d} t}=\frac{1}{2}\left(\frac{\mathrm{d}_{+} \boldsymbol{X}+\mathrm{d}_{-} \boldsymbol{X}}{\mathrm{d} t}\right)-\frac{\mathrm{i}}{2}\left(\frac{\mathrm{d}_{+} \boldsymbol{X}-\mathrm{d}_{-} \boldsymbol{X}}{\mathrm{d} t}\right) \\
& =\frac{\boldsymbol{V}_{+}+\boldsymbol{V}_{-}}{2}-\mathrm{i} \frac{\boldsymbol{V}_{+}-\boldsymbol{V}_{-}}{2} \\
& =\frac{1}{2}\left[\left(\boldsymbol{v}_{+}+\boldsymbol{v}_{-}\right)+\left(\boldsymbol{u}_{+}+\boldsymbol{u}_{-}\right)\right] \\
& -\frac{\mathrm{i}}{2}\left[\left(\boldsymbol{v}_{+}-\boldsymbol{v}_{-}\right)+\left(\boldsymbol{u}_{+}-\boldsymbol{u}_{-}\right)\right]=\boldsymbol{V}-\mathrm{i} \boldsymbol{U}
\end{aligned}
$$

with

$$
\begin{aligned}
& \boldsymbol{V}=\frac{\boldsymbol{V}_{+}+\boldsymbol{V}_{-}}{2}=\frac{1}{2}\left[\left(\boldsymbol{v}_{+}+\boldsymbol{v}_{-}\right)+\left(\boldsymbol{u}_{+}+\boldsymbol{u}_{-}\right)\right], \\
& \boldsymbol{U}=\frac{\boldsymbol{V}_{+}-\boldsymbol{V}_{-}}{2}=\frac{1}{2}\left[\left(\boldsymbol{v}_{+}-\boldsymbol{v}_{-}\right)+\left(\boldsymbol{u}_{+}-\boldsymbol{u}_{-}\right)\right] .
\end{aligned}
$$


The real part, $\boldsymbol{V}$, of the complex speed, $\hat{\boldsymbol{V}}$, represents the standard classical speed, which is differentiable and independent of resolution, while the imaginary part, $\boldsymbol{U}$, is a new quantity arising from fractality, which is non-differentiable and resolution-dependent;

(vii) "in order to account for the infinity of geodesics in the bundle, for their fractality and for the two values of the derivative which all come from the non-differentiable geometry of the space-time continuum, one therefore adopts a generalized statistical fluid like description, where instead of a classical deterministic speed or of a classical fluid speed field, one uses a doublet of fractal functions of space coordinates and time which are also explicit functions of resolution time" [9-11]. Thus, the average values of the quantities must be considered in the previously mentioned sense. Particularly, the average of $\mathrm{d}_{ \pm} \boldsymbol{X}$ is

$$
\left\langle\mathrm{d}_{ \pm} \boldsymbol{X}\right\rangle=\mathrm{d}_{ \pm} \boldsymbol{x}
$$

with

$$
\left\langle\mathrm{d}_{ \pm} \boldsymbol{\xi}\right\rangle=0
$$

(viii) in such an interpretation, the "particles", are identified with the geodesics themselves. As a consequence, any measurement is interpreted as a sorting out (or selection) of the geodesics by the measuring device [5, 9-11, 13-22].

Let us now consider a fractal function, $f(\boldsymbol{X}, t)$ and its Taylor expansion up to the second order term (the second approximation of the fractal motion). In this case, for the points on the fractal, based on the non-differentiability specified through the properties (i)-(viii) it results that the motion can be described by the Nottale fractal operator [56]:

$$
\frac{\hat{\partial}}{\partial t}+\hat{\boldsymbol{V}} \nabla-\mathrm{i} D(\mathrm{~d} t)^{\left(2 / D_{\mathrm{F}}\right)-1} \Delta .
$$

A generalization of this procedure for the third order approximation of the fractal motion is given by the fractal operator [55]:

$$
\begin{aligned}
\frac{\hat{\partial}}{\partial t} & =\frac{\partial}{\partial t}+\hat{\boldsymbol{V}} \cdot \nabla-\mathrm{i} D(\mathrm{~d} t)^{\left(2 / D_{\mathrm{F}}\right)-1} \Delta \\
& +\frac{\sqrt{2}}{3} D \sqrt{D}(\mathrm{~d} t)^{\left(3 / D_{\mathrm{F}}\right)-1} \nabla^{3} .
\end{aligned}
$$

Details on the methodology of obtaining this operator can be found in [55] (Eq. 17, p. 1564).

We now apply the principle of scale covariance, and postulate that the passage from classical (differentiable) mechanics to the "fractal" mechanics which is considered here can be implemented by replacing the standard time derivative $\mathrm{d} / \mathrm{d} t$ by the complex operator $\hat{\partial} / \partial t$ (this result is a generalization of the principle of scale covariance given by Nottale in $[5,9-11,15-22])$. As a consequence, we are now able to write the equation of geodesics (a generalization of the first Newton principle) in a fractal space-time under its covariant form

$$
\frac{\hat{\partial} \hat{\boldsymbol{V}}}{\partial t}=\frac{\partial \hat{\boldsymbol{V}}}{\partial t}+(\hat{\boldsymbol{V}} \cdot \nabla) \hat{\boldsymbol{V}}-\mathrm{i} D(\mathrm{~d} t)^{\left(2 / D_{\mathrm{F}}\right)-1} \Delta \hat{\boldsymbol{V}}
$$

$$
+\frac{2 \sqrt{2}}{3} D \sqrt{D}(\mathrm{~d} t)^{\left(3 / D_{\mathrm{F}}\right)-1} \nabla^{3} \hat{\boldsymbol{V}}=0 .
$$

This means that the global complex acceleration field, $\hat{\partial} \boldsymbol{V} / \partial t$, depends on the local complex acceleration field, $\partial_{t} \hat{\boldsymbol{V}}$, on the non-linearity (convective) term, $(\hat{\boldsymbol{V}} \cdot \nabla) \hat{\boldsymbol{V}}$, on the dissipative term, $\Delta \hat{\boldsymbol{V}}$, and on the dispersive one, $\nabla^{3} \hat{\boldsymbol{V}}$. Moreover, the behavior of a fractal fluid is of viscoelastic or of hysteretic type which means that the fractal space-time has memory. Such a result is in agreement with the opinion given in [57]: the fractal fluid can be described by the Kelvin-Voight or Maxwell rheological model with the aid of complex quantities e.g. the complex speed field, the complex acceleration field etc.

\section{The dissipative approximation of the extended scale relativity model. The expression of the fractal potential}

In the dissipative approximation of the ESR the relation (15) becomes a Navier-Stokes type equation [41]:

$$
\frac{\partial \hat{\boldsymbol{V}}}{\partial t}=\frac{\partial \hat{\boldsymbol{V}}}{\partial t}+(\hat{\boldsymbol{V}} \cdot \nabla) \hat{\boldsymbol{V}}-\mathrm{i} D(\mathrm{~d} t)^{\left(2 / D_{\mathrm{F}}\right)-1} \Delta \hat{\boldsymbol{V}}=0
$$

with an imaginary viscosity coefficient

$$
\bar{\eta}=\mathrm{i} D(\mathrm{~d} t)^{\left(2 / D_{\mathrm{F}}\right)-1} .
$$

If the motions of the fractal fluid are irrotational, i.e. $\nabla \times \hat{\boldsymbol{V}}=0$, we can choose $\hat{\boldsymbol{V}}$ of the form

$$
\hat{\boldsymbol{V}}=-2 \mathrm{i} D(\mathrm{~d} t)^{\left(2 / D_{\mathrm{F}}\right)-1} \nabla \ln \psi
$$

with $\psi$ the scalar potential of the complex speed. By substituting (17) in (16), up to an arbitrary phase factor which may be set to zero by a suitable choice of the phase origin, a Schrödinger type equation results

$$
D^{2} \mathrm{~d} t^{\left(4 / D_{\mathrm{F}}\right)-2} \Delta \psi+\mathrm{i} D \mathrm{~d} t^{\left(2 / D_{\mathrm{F}}\right)-1} \frac{\partial \psi}{\partial t}=0 .
$$

For $D=\hbar / 2 m_{0}$ and $D_{\mathrm{F}}=2$, i.e. for movements on the Peano's curves [9-11] at the Compton scale, Eq. (18) reduces to the usual Schrödinger equation

$$
\frac{\hbar^{2}}{2 m_{0}} \Delta \psi+\mathrm{i} \hbar \frac{\partial \psi}{\partial t}=0 \text {. }
$$

For $\psi=\sqrt{\rho} \mathrm{e}^{\mathrm{i} S}$, with $\sqrt{\rho}$ the amplitude and $S$ the phase of $\psi$, the complex speed field (10), using (17) takes the explicit form

$$
\begin{aligned}
\hat{\boldsymbol{V}} & =2 D(\mathrm{~d} t)^{\left(2 / D_{\mathrm{F}}\right)-1} \nabla S-\mathrm{i} D(\mathrm{~d} t)^{\left(2 / D_{\mathrm{F}}\right)-1} \nabla \ln \rho, \\
\boldsymbol{V} & =2 D(\mathrm{~d} t)^{\left(2 / D_{\mathrm{F}}\right)-1} \nabla S \\
\boldsymbol{U} & =D(\mathrm{~d} t)^{\left(2 / D_{\mathrm{F}}\right)-1} \nabla \ln \rho .
\end{aligned}
$$

By substituting $(20 \mathrm{a}-\mathrm{c})$ in $(16)$ and separating the real and the imaginary parts, up to an arbitrary phase factor which may be set at zero by a suitable choice of the phase of $\psi$, we obtain

$$
m_{0}\left(\frac{\partial \boldsymbol{V}}{\partial t}+(\boldsymbol{V} \cdot \nabla) \boldsymbol{V}\right)=-\nabla Q
$$




$$
\frac{\partial \rho}{\partial t}+\nabla \cdot(\rho V)=0
$$

with $Q$ the fractal potential

$$
\begin{aligned}
Q & =-2 m_{0} D^{2}(\mathrm{~d} t)^{\left(4 / D_{\mathrm{F}}\right)-2} \frac{\Delta \sqrt{\rho}}{\sqrt{\rho}} \\
& =-\frac{m_{0} \boldsymbol{U}^{2}}{2}-m_{0} D \mathrm{~d} t^{\left(2 / D_{\mathrm{F}}\right)-1} \nabla \cdot \boldsymbol{U}
\end{aligned}
$$

The idea of fractal potential appears for the first time in [58] as new potential term named "Bohm potential". Particularly, for movements on the Peano's curves at the Compton scale, the fractal potential can be assimilated with "Bohm potential". In SR theory, the fractal potential comes, logically, from the non-differentiability of the fractal space-time.

For $D=\hbar / 2 m_{0}$ and $D_{\mathrm{F}}=2$, i.e. for movements on Peano's curves at the Compton scale, the fractal potential becomes the usual quantum potential

$$
Q_{Q M}=-\frac{\hbar^{2}}{2 m_{0}} \frac{\Delta \sqrt{\rho}}{\sqrt{\rho}}=-\frac{m_{0} \boldsymbol{U}^{2}}{2}-\frac{\hbar}{2} \nabla \cdot \boldsymbol{U} .
$$

\section{The dispersive approximation of the extended scale relativity model. Type I superconductivity}

In the dispersive approximation of the ESR the relation (15) becomes a Korteweg-de Vries type equation

$$
\begin{aligned}
& \frac{\partial \hat{\boldsymbol{V}}}{\partial t}=\frac{\partial \hat{\boldsymbol{V}}}{\partial t}+(\hat{\boldsymbol{V}} \cdot \nabla) \hat{\boldsymbol{V}} \\
& \quad+\frac{2 \sqrt{2}}{3} D \sqrt{D}(\mathrm{~d} t)^{\left(3 / D_{\mathrm{F}}\right)-1} \nabla^{3} \hat{\boldsymbol{V}}=0 .
\end{aligned}
$$

This means that in the fractal space-time, the dissipative effects can be neglected compared with the convective and dispersive ones.

If the motions of the fractal fluid are irrotational, we can choose for the complex speed field the expression (17). The real part of the complex speed field is (20b) and the imaginary one is (20c). Particularly, for coherent, syncron motions

$$
\boldsymbol{V}=\boldsymbol{U}
$$

and the complex speed field takes the equivalent forms

$$
\begin{aligned}
\hat{\boldsymbol{V}} & =2 D(\mathrm{~d} t)^{\left(\frac{2}{D_{\mathrm{F}}}\right)-1} \nabla S-2 \mathrm{i} D(\mathrm{~d} t)^{\left(\frac{2}{D_{\mathrm{F}}}\right)-1} \nabla S \\
& =D(\mathrm{~d} t)^{\left(\frac{2}{D_{\mathrm{F}}}\right)-1} \nabla \ln \rho-\mathrm{i} D(\mathrm{~d} t)^{\left(\frac{2}{D_{\mathrm{F}}}\right)-1} \nabla \ln \rho \\
& =2 \eta \nabla S=\eta \nabla \ln \rho, \\
\eta & =D(\mathrm{~d} t)^{\left(\frac{2}{D_{\mathrm{F}}}\right)-1}(1-\mathrm{i}) .
\end{aligned}
$$

It results, both through the complex speed field, $\hat{\boldsymbol{V}}$, and the structure coefficient, $\eta$, the rheological behavior of the fractal fluid.

Now, after the separation of real and imaginary parts, Eq. (23) becomes

$$
\frac{\partial \boldsymbol{V}}{\partial t}+\nabla\left(\frac{\boldsymbol{V}^{2}}{2}-\frac{\boldsymbol{U}^{2}}{2}\right)
$$

$$
\begin{aligned}
&+ \frac{2 \sqrt{2}}{3} D \sqrt{D}(\mathrm{~d} t)^{\left(3 / D_{\mathrm{F}}\right)-1} \nabla^{3} \boldsymbol{V}=0, \\
& \frac{\partial \boldsymbol{U}}{\partial t}+\nabla(\boldsymbol{V} \cdot \boldsymbol{U}) \\
&+\frac{2 \sqrt{2}}{3} D \sqrt{D}(\mathrm{~d} t)^{\left(3 / D_{\mathrm{F}}\right)-1} \nabla^{3} \boldsymbol{U}=0 .
\end{aligned}
$$

In the one-dimensional differentiable case, i.e. for $\rho=$ const, Eqs. (26a,b), with the dimensionless parameters

$$
\begin{aligned}
& \tau=\omega t, \quad \xi=k x, \quad \theta=\xi-v_{\mathrm{ph}} \tau, \\
& w(\theta)=\frac{V}{V_{0}}
\end{aligned}
$$

and the normalizing conditions

$$
\frac{k V_{0}}{6 \omega}=\frac{2 \sqrt{2}}{3} D \sqrt{D}(\mathrm{~d} t)^{\left(3 / D_{\mathrm{F}}\right)-1} \frac{k^{3}}{\omega}=1,
$$

by double integration becomes

$$
\frac{1}{2} w^{\prime 2}=F(w)=-\left(w^{3}-\frac{v_{\mathrm{ph}}}{2} w^{2}-g w-h\right)
$$

with $g, h$ two integration constants and $v_{\mathrm{ph}}$ the normalized phase velocity.

From here, through the fractalization of the differentiable part of the complex speed field which improves the substitutions

$$
w=\frac{v_{\mathrm{ph}}}{4} f^{2}, \quad \frac{2 \theta}{\sqrt{v_{\mathrm{ph}}}}=\mathrm{i} \beta
$$

and the restriction $h=0$, Eq. (29) becomes a GinzburgLandau (GL) type equation [59]

$$
\frac{\mathrm{d}^{2} f}{\mathrm{~d} \beta}=f^{3}-f .
$$

Multiplying both sides of Eq. (31) by $\mathrm{d} f / \mathrm{d} \beta$, and performing integration over $\beta$ we obtain

$$
\frac{\mathrm{d} f}{\mathrm{~d} \beta}=\sqrt{\frac{1}{2} f^{4}-f^{2}+C},
$$

where

$$
C=\left[\left(\frac{\mathrm{d} f}{\mathrm{~d} \beta}\right)^{2}-\frac{1}{2} f^{4}+f^{2}\right]_{\beta=\beta_{0}} .
$$

Equation (33) is obviously a restriction imposed on the order parameter, $f$, showing the boundary conditions. A further integration of Eq. (32) leads to

$$
\frac{\beta-\beta_{0}}{\sqrt{2}}=\int_{0}^{f} \frac{\mathrm{d} f}{\sqrt{\left(f_{1}^{2}-f^{2}\right)\left(f_{2}^{2}-f^{2}\right)}},
$$

where

$$
f_{1,2}^{2}=1 \mp \sqrt{1-2 C} .
$$

By the change of variable $\phi=f / f_{1}$, the integral (34) becomes

$$
f_{2} \frac{\beta-\beta_{0}}{\sqrt{2}}=\int_{0}^{f / f_{1}} \frac{\mathrm{d} \phi}{\sqrt{\left(1-\phi^{2}\right)\left(1-s^{2} \phi^{2}\right)}},
$$

where we made the notation

$$
s \equiv f_{1} / f_{2} \text {. }
$$

Writing $f_{1}$ and $f_{2}$ in terms of $s, f_{1}^{2}=\left(2 s^{2} / 1+s^{2}\right)$, $f_{2}^{2}=\left(2 / 1+s^{2}\right)$, the integral (36) becomes 


$$
\frac{\beta-\beta_{0}}{\sqrt{1+s^{2}}}=\int_{0}^{\phi_{1}} \frac{\mathrm{d} \phi}{\sqrt{\left(1-\phi^{2}\right)\left(1-s^{2} \phi^{2}\right)}},
$$

where the superior limit is

$$
\phi_{1}=f \sqrt{\frac{1+s^{2}}{2 s^{2}}} .
$$

The integral (38) can be solved in the terms of the Jacobian elliptic functions, $s n(u ; s)$ of argument $u$ and modulus $s[60]$. The class of solutions results

$$
f=\sqrt{\frac{2 s^{2}}{1+s^{2}}} \operatorname{sn}\left(\frac{\beta-\beta_{0}}{\sqrt{1+s^{2}}} ; s\right)
$$

In the limit $s \rightarrow 0,(39)$ can be approximated as

$$
f=\sqrt{\frac{2 s^{2}}{1+s^{2}}} \sin \left(\frac{\beta-\beta_{0}}{\sqrt{1+s^{2}}}\right) \rightarrow 0,
$$

while for $s \rightarrow 1$ one obtains the kink solution

$$
f=\sqrt{\frac{2 s^{2}}{1+s^{2}}} \tanh \left(\frac{\beta-\beta_{0}}{\sqrt{1+s^{2}}}\right) .
$$

It is well known that the GL model also supports two solutions: zero and hyperbolic tangent which correspond to the Cooper pair [61]. Thus, we can build a field theory with spontaneous symmetry breaking: the kink solution spontaneously breaks the vacuum symmetry by tunneling and generates Cooper type pairs [62].

We are able now to get the expressions of some superconducting parameters. Thus, from (38), by integration and using the relation which defines the relative coherence length [62-64], it results

$$
\xi_{\mathrm{r}}=\left(1+s^{2}\right)^{1 / 2} K(s),
$$

where $K(s)$ is the complete elliptic integral of the first kind [60],

$$
K(s)=\int_{0}^{\pi / 2}\left(1-s^{2} \sin ^{2} \phi\right)^{-1 / 2} \mathrm{~d} \phi .
$$

The relative critical speed

$$
v_{\mathrm{r}}=\left(1+s^{2}\right)^{-1 / 2} K^{-1}(s)
$$

and the relative pair breaking time

$$
\tau_{\mathrm{r}}=\left(1+s^{2}\right) K^{2}(s)
$$

are deduced from (42) and the relations which define these parameters [62-64].

In the $1 \mathrm{D}$ case, we can express the relative charge carrier concentration as

$$
n_{\mathrm{r}}=|f|^{2}=\frac{2 s^{2}}{1+s^{2}} s n^{2}\left(\frac{\beta-\beta_{0}}{\sqrt{1+s^{2}}} ; s\right) .
$$

In order to establish a direct connection with experimental observations in the physical systems, the acquired spatial dependence of $n_{\mathrm{r}}$ can be cancelled by averaging $|f|^{2}$ on a period, $2 K$ :

$$
n_{\mathrm{r}}=\frac{2}{1+s^{2}}\left[1-\frac{E(s)}{K(s)}\right]
$$

where $E(k)$ is the complete elliptic integral of the second kind of modulus $s[60]$ :

$$
E(s)=\int_{0}^{\pi / 2}\left(1-s^{2} \sin ^{2} \phi\right)^{1 / 2} \mathrm{~d} \phi .
$$

By using (45) and the London definition of the pene- tration depth [62-64], we obtain the relative penetration depth in the case of spatial gradients

$$
\lambda_{\mathrm{r}}=\left\{\frac{2}{1+s^{2}}\left[1-\frac{E(s)}{K(s)}\right]\right\}^{-1 / 2} .
$$

For the relative critical field, using the definition of this parameter [62-64] we derive the expression

$$
B_{\mathrm{r}}=\frac{2}{1+s^{2}} \sqrt{2\left(1-\frac{E(s)}{K(s)}\right)} .
$$

The relative critical current density

$$
j_{\mathrm{r}}=\frac{2}{\left(1+s^{2}\right)^{3 / 2}}\left[\frac{1}{K(s)}-\frac{E(s)}{K^{2}(s)}\right]
$$

is obtained from (44) and (47) and the expression which defines $j_{\mathrm{r}}[62-64]$.

Taking into account that all the "superconducting" parameters suffer a discontinuity for $s=1$ (as can be seen in Fig. 1a-g), one can admit the following functional dependence of the modulus of the elliptic function on the reduced temperature:

$$
s \equiv t=T / T_{\mathrm{C}} .
$$

To avoid the confusion and to obtain the exact temperature dependences of the superconducting parameters, we will admit adequate normalizations of these parameters and the restriction $s<1$. Thus, the dependence on $t$ of the coherence length is

$$
\xi(t) / \xi(0)=(2 / \pi)\left(1+t^{2}\right)^{1 / 2} K(t) .
$$

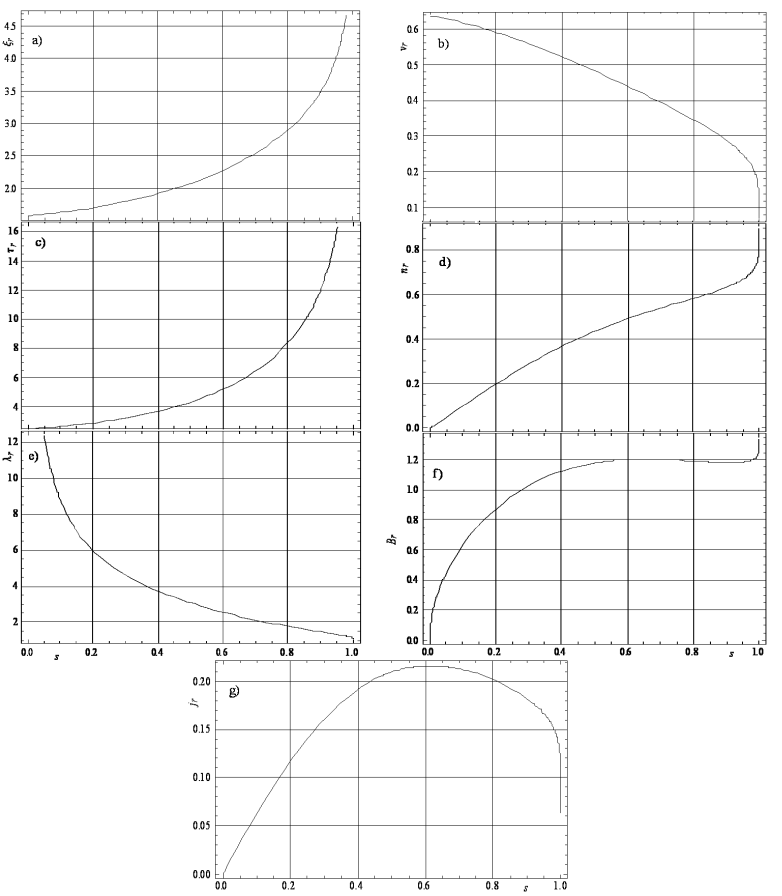

Fig. 1. The $s$ dependence of the (a) relative coherence length, (b) relative critical speed, (c) relative pair breaking time, (d) average relative concentration, (e) relative penetration depth, (f) relative critical field, (g) relative critical current density. 


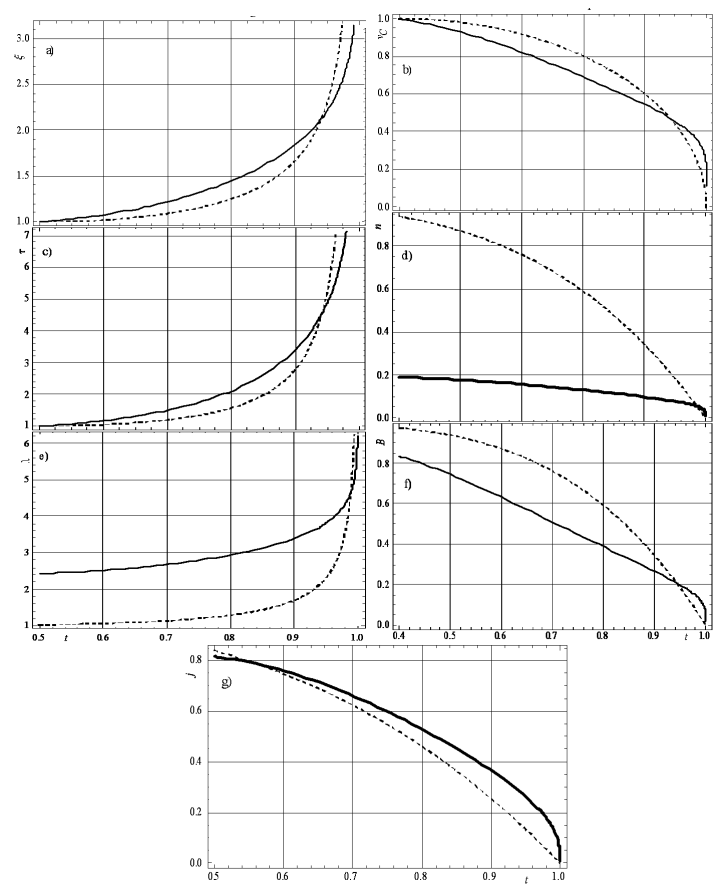

Fig. 2. The comparison between the reduced temperature dependence of the superconductor parameters as given by Eqs. (53)-(55) and (57)-(60) (continuous line) with the one given by BCS or other theories (dashed line): (a) the relative coherence length, (b) the relative critical speed, (c) the relative pair breaking time, (d) the average relative concentration, (e) the relative penetration depth, (f) the relative critical field, (g) the relative critical current density.

Figure 2a shows the relation (53) in comparison with the one given by the BCS theory [62], i.e. $\xi(t) / \xi(0)=\left(1-t^{2}\right)^{-1 / 2}$ (dashed line). In the relation $(53)$

$$
K(t)=\int_{0}^{\pi / 2}\left(1-t^{2} \sin ^{2} \phi\right)^{-1 / 2} \mathrm{~d} \phi .
$$

The dependence on $t$ of the critical speed is

$$
\left(v_{\mathrm{c}}(t) / v_{\mathrm{c}}(0)\right)=(\pi / 2)\left(1+t^{2}\right)^{-1 / 2} K^{-1}(t) .
$$

In Fig. 2b one can see the comparison between the (54) dependence and the one given by the BCS theory [62] i.e. $v_{\mathrm{c}}(t) / v_{\mathrm{c}}(0)=\left(1-t^{2}\right)^{1 / 2}$ (dashed line).

The dependence on $t$ of the pair breaking time is

$$
\tau(t) / \tau(0)=\left(4 / \pi^{2}\right)\left(1+t^{2}\right) K^{2}(t) .
$$

Figure 2c presents together the dependence (55) and the one given by BCS theory [62], i.e. $\tau(t) / \tau(0)=\left(1-t^{2}\right)^{-1}$ (dashed line).

Comparing with the BCS theory [62], similar behaviors of the coherence length (Fig. 2a), critical speed (Fig. 2b) and pair breaking time (Fig. 2c) can be observed.

In order to get the dependence of the concentration on $t$ one admits first that

$$
n(t) / n(0) \approx[\xi(0) / \xi(t)]^{2} f^{2}=3.7\left(1+t^{2}\right)^{-1} K^{-2}(t) f^{2} .
$$

By inserting (47) in (56) one finds

$$
n(t) / n(0)=3.7\left(1+t^{2}\right)^{-2} K^{-2}(t)\{1-[E(t) / K(t)]\} .
$$

The concordance with the experimental data described by $n(t) / n(0)=\left(1-t^{4}\right)^{1 / 2}$ (dashed line) is relatively good in the limits $0.5<t<1$, as can be seen in Fig. 2d.

The reduced temperature dependence of the penetration depth

$$
\lambda(t) / \lambda(0)=0.55\left(1+t^{2}\right) K(t)\{1-[E(t) / K(t)]\}^{-1 / 2}
$$

is in agreement with the data of the bifluid model $\lambda(t) / \lambda(0)=\left(1-t^{4}\right)^{-1 / 2}$ (dashed line - Fig. 2e, for $0.5<t<1$.

In the case of the critical field,

$$
\begin{aligned}
& B_{\mathrm{c}}(t) / B_{\mathrm{c}}(0)=7.67\left(1+t^{2}\right)^{-2} K^{-2}(t) \\
& \quad \times\{1-[E(t) / K(t)]\}^{1 / 2}
\end{aligned}
$$

the agreement with the experimental data, which is well described by $B_{\mathrm{c}}(t) / B_{\mathrm{c}}(0)=1-t^{2}[62]$ (dashed line), can be seen in Fig. 2f, where Eq. (59) fits data for $0.4<t<1$.

The dependence on $t$ of the critical current density is

$$
\begin{aligned}
& j_{\mathrm{c}}(t) / j_{\mathrm{c}}(0)=26.7\left(1+t^{2}\right)^{-3 / 2} K^{-3}(t) \\
& \quad \times\{1-[E(t) / K(t)]\} .
\end{aligned}
$$

In Fig. $2 \mathrm{~g}$ one can see the comparison between the (61) dependence and the one given by the BCS theory [62], i.e. $j_{\mathrm{c}}(t) / j_{\mathrm{c}}(0)=\left(1-t^{2}\right)^{1 / 2}\left(1-t^{4}\right)^{1 / 2}$ (dashed line). The concordance is good in the limits $0.5<t<1$.

Summarizing, we would emphasize that the derived expressions of the superconducting parameters $(n(t), \lambda(t)$, $\left.B_{\mathrm{c}}(t), j_{\mathrm{c}}(t)\right)$ are amendable to experimental verifications in the range about $0.5<t<1$. At the same time, $\xi(t)$, $v_{\mathrm{c}}(t), \tau(t)$ have similar behaviors for $0<t<1$.

Since the general solution of GL equation is (39), the superconductivity is controlled by means of the normalized fractal potential,

$$
\begin{aligned}
& Q(\eta, s)=-\frac{1}{f} \frac{\mathrm{d}^{2} f}{\mathrm{~d} \eta^{2}}=\left(1-f^{2}\right) \\
& \quad=\frac{1-s^{2}}{1+s^{2}}+\frac{2 s^{2}}{1+s^{2}} c n^{2}\left(\frac{\beta-\beta_{0}}{\sqrt{1+s^{2}}} ; s\right)
\end{aligned}
$$

also through cnoidal oscilation modes [46]. Thus, for $s=0$ or $s \rightarrow 0$ it results the non-quasi-autonomous regime (of linear wave or of wave packet type),

$$
\begin{aligned}
& Q(\eta, s=0, s \rightarrow 0) \\
& \quad=\frac{1-s^{2}}{1+s^{2}}+\frac{2 s^{2}}{1+s^{2}} \cos ^{2}\left(\frac{\beta-\beta_{0}}{\sqrt{1+s^{2}}} ; s\right)
\end{aligned}
$$

and for $s=1$ or $s \rightarrow 1$ the quasi-autonomous regime (of soliton or of soliton packet type),

$$
\begin{aligned}
& Q(\eta, s=1, s \rightarrow 1) \\
& \quad=\frac{1-s^{2}}{1+s^{2}}+\frac{2 s^{2}}{1+s^{2}} \operatorname{sech}^{2}\left(\frac{\beta-\beta_{0}}{\sqrt{1+s^{2}}} ; s\right) .
\end{aligned}
$$


The normalized fractal potential (61) takes a very simple expression which is directly proportional to the density of states of the Cooper type pairs. When the density of states of the Cooper type pairs, $f^{2}$, becomes zero, the fractal potential takes a finite value, $Q=1$. The fractal fluid is normal (it works in a non-quasi-autonomous regime) and there are no coherent structures (the Cooper type pairs) in it. When $f^{2}$ becomes 1 , the fractal potential is zero, i.e. the entire quantity of energy of the fractal fluid is transferred to its coherent structures, i.e. to the superconducting type pairs. Then the fractal fluid becomes "superconducting" (it works in a quasi-autonomous regime). Therefore, one can assume that the energy from the fractal fluid can be stocked by transforming all the environment's entities into coherent structures (Cooper type pairs) and then "freezing" them. The "superconducting" fluid acts as an energy accumulator through the fractal potential (61).

\section{Conclusions}

The main conclusions of the present paper are the following:

(i) Considering that the microparticle movements take place on fractal curves, the role of the fractal potential in the system dynamics through an extension of scale relativity theories is analyzed;

(ii) The absence of the dispersion implies a generalized Navier-Stokes type equation. From here, for the irrotational movement, both Schrödinger-type equation and hydrodynamic model of the scale relativity theory result. Thus, the fractal potential comes from the non-differentiability of the fractal space-time;

(iii) The absence of dissipation implies a generalized Korteweg-de Vries type equation. In such conjecture, through the fractalization of differentiable part of the complex speed field, the fractal potential controls by means of coherence the system dynamics. The status of the fractal potential is detailed for type-I superconductivity. So, it resulted that: (iii ${ }_{1}$ ) the type-I superconductivity is achieved by one-dimensional cnoidal oscillation modes of the complex speed field; (iii ${ }_{2}$ ) for different coherence degrees, the one-dimensional cnoidal speed oscillation modes contain the one-dimensional harmonic waves, the one-dimensional waves packet, the one-dimensional solitons packet and the one-dimensional soliton. The first two subsequences describe the non-autonomous regime for type-I superconductor, i.e. an ordinary fractal fluid behavior, while the last ones describe the quasi-autonomous regime for type-I superconductor, i.e. a coherent fractal fluid behavior by means of Cooper-type pairs. ( $\mathrm{iii}_{3}$ ) We can build a field theory with spontaneous symmetry breaking corresponding to the normal conductor-superconductor phase transition. The fractal kink spontaneously breaks the "vacuum symmetry" of the fractal fluid by tunneling, and generates coherent structures (the Cooper pairs). Moreover, the fractal fluid acts as an energy accumulator through the fractal potential (fractal soliton); ( $\left.\mathrm{iii}_{4}\right)$ the temperature dependences of the superconducting parameters, are obtained: the coherence length, the critical speed, the pair breaking time, the charge carrier concentration, the penetration depth, the critical field, the critical current density.

We note that the fractal (stochastic) processes $[5,6]$ contained in the operator (14) with $D_{\mathrm{F}} \neq 2$ are known as "anomalous diffusion" (sub-diffusion for $D_{\mathrm{F}}<2$ and super-diffusion for $D_{\mathrm{F}}>2$ ). Many expect that the "Fokker-Planck equations" for anomalous diffusion do not have the form of the ordinary diffusion equation. Indeed, it is well-known that the "Fokker-Planck equations" for anomalous diffusion have the form of the fractional derivative equations, and the equations are called fractional Fokker-Planck equations [47-54]. However, we are able to use the non-fractional derivative equation in the study of anomalous diffusion as it results from [2-5].

\section{Acknowledgments}

The authors wish to thank the referees for the pertinent observations.

\section{References}

[1] B. Mandelbrot, The Fractal Geometry of Nature, Freeman, San Francisco 1982.

[2] E. Nelson, Quantum Fluctuations, Princeton Univ. Press, New York 1985.

[3] J. Feder, A. Aharony, Fractals in Physics, NorthHolland, Amsterdam 1990.

[4] J.F. Gouyet, Physique et Structures Fractals, Masson, Paris 1992.

[5] L. Nottale, Fractal Space-Time and Microphysics: Towards a Theory of Scale Relativity, World Scientific, Singapore 1993.

[6] M.S. El Naschie, O.E. Roessler, I. Prigogine, Quantum Mechanics, Diffusion and Chaotic Fractal, Elsevier, Oxford 1995.

[7] J. Argyris, C. Ciubotariu, G. Mattutis, Chaos, Solitons Fractals 12, 1 (2001).

[8] Space-time Physics and Fractality, Eds. P. Weibel, G. Ord, G. Rössler, Springer, Vienna 2005.

[9] L. Nottale, The Universe and the light. Classical cosmology and gravitational mirage, Flammarion, Paris 1993 (in French).

[10] L. Nottale, Relativity in all its forms: from movements to scale changes, Hachette, Paris 1998 (in French).

[11] L. Nottale, J. Chaline, P. Grou, The trees of evolution, Hachette, Paris 2000 (in French).

[12] C.P. Cristescu, Nonlinear Dynamics and Chaos in Science and Engineering, Academy Publishing House, Bucharest 2008.

[13] L. Nottale, in: Proc. First Int. Conf. on the Evolution and Development of the Universe, Paris 2008, p. 15.

[14] L. Nottale, Ch. Auffray, Prog. Biophys. Mol. Biol. 97, 115 (2008).

[15] L. Nottale, J. Phys. A, Math. Theor. 42, 275306 (2009). 
[16] L. Nottale, Chaos, Solitons Fractals 9, 1051 (1998).

[17] L. Nottale, Chaos, Solitons Fractals 10, 459 (1999).

[18] L. Nottale, Chaos, Solitons Fractals 16, 539 (2003).

[19] D. Da Rocha, L. Nottale, Chaos, Solitons Fractals 16, 565 (2003).

[20] L. Nottale, Chaos, Solitons Fractals 25, 797 (2005).

[21] L. Nottale, M.N. Célérier, T. Lehner, J. Math. Phys. 47, 032303 (2006).

[22] M.N. Célérier, L. Nottale, J. Phys. A, Math. Gen. 37, 931 (2004).

[23] D. Bohm, Phys. Rev. 85, 166 (1952).

[24] D. Bohm, Phys. Rev. 85, 180 (1952).

[25] D. Bohm, Phys. Rev. 89, 458 (1953).

[26] D. Bohm, B.J. Hihey, Phys. Rev. Lett. 55, 2511 (1985).

[27] D. Bohm, B.J. Hihey, The Undivided Universe: An Ontological Interpretation of Quantum Theory, Ronthege Kegan Paul, London 1993.

[28] P.R. Holland, The Quantum Theory of Motion, Cambridge University Press, Cambridge 1993.

[29] M. Agop, O. Niculescu, A. Timofte, L. Bibire, A.S. Ghenadi, A. Nicuta, C. Nejneru, G.V. Munceleanu, Int. J. Theor. Phys., DOI: 10.1007/s10773-010$-0330-5$.

[30] C.Gh. Buzea, C. Bejinariu, C. Boris, P.V. Vizureanu, M. Agop, Int. J. Nonlin. Sci. Numer. Simul. 10, 1399 (2009).

[31] M. Agop, G.V. Munceleanu, O. Niculescu, T. Dandu-Bibire, Phys. Scr. 82, 015010 (2010).

[32] P.D. Ioannou, P. Nica, V. Paun, P. Vizureanu, M. Agop, Phys. Scr. 78, 065101 (2008).

[33] C.Gh. Buzea, I. Rusu, V. Bulancea, Gh. Badarau, V.P. Paun, M. Agop, Phys. Lett A 374, 2757 (2010).

[34] M. Agop, P.E. Nica, P.D. Ioannou, A. Antici, V.P. Paun, Euro Phys. J. D 49, 239 (2008).

[35] M. Agop, P.E. Nica, S. Gurlui, C. Focsa, V.P. Paun, M. Colotin, Euro Phys. J. D 10.11.40/epjd/ e2009_00304-5.

[36] S. Gurlui, M. Agop, P. Nica, M. Ziskind, C. Focsa, Phys. Rev. E 78, 026405 (2008).

[37] O. Niculescu, D.G. Dimitriu, V.P. Paun, P.D. Matasaru, D. Scurtu, M. Agop, Phys. Plasmas 17, 042305 (2010).

[38] S. Gurlui, M. Agop, M. Strat, G. Strat, S. Bacaita, A. Cerepaniuc, Phys. Plasmas 13, 063503 (2006).

[39] M. Agop, P. Nica, M. Gartu, Gen. Relativ. Gravit. 401, 35 (2008).

[40] P. Nica, P. Vizureanu, M. Agop, S. Gurlui, C. Focsa, N. Forna, P.D. Ioannou, Z. Borsos, Jpn. J. Appl. Phys. 48, 066001 (2009).
[41] C. Stan, C.P. Cristescu, D. Alexandroaie, M. Agop, Chaos Solitons Fractals 41, 727 (2009).

[42] M. Agop, C. Radu, T. Bontas, Chaos Solitons Fractals 38, 1243 (2008).

[43] C.P. Cristescu, B. Mereu, C. Stan, M. Agop, Chaos Solitons Fractals 40, 975 (2009).

[44] L. Delle Site, Europhys Lett. 57, 20 (2002).

[45] L. Delle Site, Physica A 313, 453 (2002).

[46] M. Colotin, G.O. Pompilian, P. Nica, S. Gurlui, V. Paun, M. Agop, Acta Phys. Pol. A 116, 157 (2009).

[47] J. Cresson, Int. J. Geometric Meth. Mod. Phys. 3, 1395 (2006).

[48] J. Cresson, J. Math. Anal. Appl. 307, 48 (2005).

[49] J. Cresson, F. Ben Adda, Chaos Solitons Fractals 19, 1323 (2004).

[50] J. Cresson, J. Math. Phys. 44, 4907 (2003).

[51] J. Cresson, Chaos Solitons Fractals 14, 553 (2002).

[52] J. Cresson, F. Ben Adda, J. Math. Anal. Appl. 262, 721 (2001).

[53] J. Cresson, J.-N. Denarie, Lecture Notes in Physics, Planat, Paris 2000.

[54] J. Cresson, F. Ben Adda, C.R. Acad. Sci. Paris 330 , 261 (2000).

[55] M. Agop, A. Harabagiu, P. Nica, Acta Phys. Pol. A 113, 1557 (2008).

[56] L. Nottale, Chaos Solitons Fractals 7, 877 (1996).

[57] D.K. Ferry, S.M. Goodnick, Transport in Nanostructures, Cambridge University Press, Cambridge 1997.

[58] L. Nottale, G. Schumacher, E.T. Lefevre, Astron. Astrophys. 361, 384 (2000).

[59] E.A. Jackson, Perspectives in Nonlinear Dynamics, Vol. I+II, Cambridge University, Cambridge 1991.

[60] F. Bowman, Introduction to Elliption Function with Applications, English University Press, London 1997.

[61] C.P. Poole, H.A. Farach, R.J. Creswick, Superconductivity, Academic Press, San Diego 1995.

[62] M. Chaichian, F.N. Nelipa, Introduction to Gauge Field Theories, Springer, Berlin 1984.

[63] J.R. Schrieffer, Theory of Superconductivity, Benjamin, New York 1964.

[64] Superconductivity, Ed. R.D. Parks, Vol. I, II, Dekker, New York 1969. 